\title{
MAL'TSEV CONDITIONS AND SPECTRA
}

\author{
WALTER TAYLOR
}

(Received 21 August 1978; revised 16 January 1979)

Communicated by R. Lidl

1980 Mathematics subject classification (Amer. Math. Soc.):08 B 05

Let $J$ be a cofinite set of positive integers which contains 1. In (1973) I proved that the following condition on a variety (equational class) $\mathscr{V}$ is Mal'tsev-definable: if $\mathfrak{U} \in \mathscr{V}$ and $\mathfrak{U}$ is finite, then $|\mathfrak{U}| \in J$. This article contains some subsidiary results, concerned mainly with a more detailed description of these Mal'tsev conditions. Many of our results arose upon considering a recent article of W. D. Neumann (1978).

To restate the above result in more detail, let us first recall that the spectrum of a variety $\mathscr{V}$ is the set of finite cardinalities of algebras in $\mathscr{V}$. Then that theorem asserts the existence, for each $J$, of a sequence $\varphi_{1}, \varphi_{2}, \varphi_{3}, \ldots$ of finite conjunctions of identities, such that Spec $\mathscr{V} \subseteq J$ if and only if some $\varphi_{i}$ holds in $\mathscr{V}$ (after suitably interpreting the operation symbols appearing in $\varphi_{i}$ as $\mathscr{V}$-terms). (These $\varphi_{i}$ are loosely known as Mal'tsev 'conditions'. It is usually also required that $\varphi_{i+1}$ should follow from $\varphi_{i}$ after suitably interpreting the operation symbols of $\varphi_{i+1}$ as $\varphi_{i}$-terms; in our case this is possible if and only if $J$ is closed under multiplication, which we will assume to be true.) Here we are concerned with the questions: Can the sequence $\varphi_{1}, \varphi_{2}, \ldots$ be taken finite? How many variables are needed for the identities appearing in this sequence? How easy is it to actually write this sequence?

Our first theorem simply states that the sequence cannot be taken finite.

THEOREM 1. 'Spec $\mathscr{V} \subseteq J$ ' is not a strong Mal'tsev condition.

This result is an immediate corollary of stronger results to be proved in Section 1.

Research supported in part by N.S.F. Grant MCS 77-01668. 
It contradicts a result 'proved' in Neumann (1978). In Section 1 we will also comment on some related false results of that article. In Section 2 we will address the second question, with this result:

THEOREM 2. ( $p$ prime.) If $J=\omega-\{p\}$, then $p$ variables are required for the Mal'tsev condition for $\operatorname{Spec} \mathscr{V} \subseteq J$.

Our third question has no precise answer, since 'easy' has no mathematical definition. But in Section 3 we will provide simple algorithms to show that writing $\varphi_{1}, \varphi_{2}, \ldots$ is just as easy as, and probably no easier than, writing a Horn sentence with spectrum $J$. This seems as close as one can come to answering Problem 4.2 of Neumann (1978), who asked for 'nice, explicit' conditions.

TheOREM 3. There exist $2^{N_{0}}$ varieties of varieties. (See Section 4 for definitions.)

The results of this article were announced in W. Taylor (1978) and (1979).

\section{Proof of Theorem 1}

The reader should have a modest familiarity with general algebra and lattice theory. We will define all notions special to the theory of Mal'tsev conditions, but even so this article will be easier to read if one has some familiarity with one of the three background papers, Taylor (1973), Neumann (1974) or Baldwin and Berman (1977), or especially its immediate predecessor, Neumann (1978).

For varieties $\mathscr{V}_{1}, \mathscr{V}_{2}, \ldots$ (not necessarily of the same similarity type), we define their product $\Pi \mathscr{V}_{i}$ to be the variety generated by all algebras of the form $\left(\prod A_{i}, F_{t}\right)_{t \in T}$, where each $A_{i}$ is the underlying set of an algebra $\mathfrak{U}_{i} \in \mathscr{V}_{i}$, and the operations $F_{t}$ are defined as follows. For every $n$ and every sequence

$$
p_{i}\left(x_{1}, \ldots, x_{n}\right)(i=1,2, \ldots)
$$

with each $p_{i}$ a derived operation of $\mathscr{V}_{i}$, we define $F:\left(\prod A_{i}\right)^{n} \rightarrow\left(\prod A_{i}\right)$ via

$$
F\left(a_{1}, \ldots, a_{n}\right)=\left\langle p_{1}\left(a_{1}^{1}, \ldots, a_{n}^{1}\right), p_{2}\left(a_{1}^{2}, \ldots, a_{n}^{2}\right), \ldots\right\rangle ;
$$

these are all the operation of $\prod_{\mathscr{V}_{i}}$. A basic result in the theory of Mal'tsev conditions (op. cit.) is that a Mal'tsev condition is strong if and only if it is preserved under the formation of products of varieties. And so Theorem 1 is an immediate corollary of the main result of this section:

Proposition 1.1. There exist varieties $\mathscr{V}_{1}, \mathscr{V}_{2}, \mathscr{V}_{3} \ldots$ such that each $\mathscr{V}_{i}$ contains no non-trivial finite algebras, but $\prod \mathscr{V}_{i}$ has a subvariety equivalent to the variety of 
bounded distributive lattices. (In particular, $\prod \mathscr{V}_{i}$ contains algebras of every finite power.)

Proof. Let $L_{i}$ be the lattice

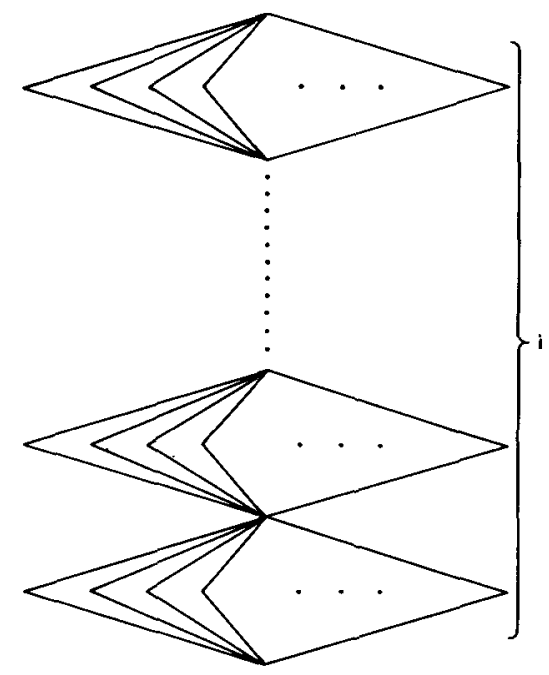

where each 'diamond' has a top and a bottom, $\aleph_{0}$ atoms, and nothing else: there are $i$ diamonds mounted vertically. Define

$$
\mathscr{V}_{i}=\operatorname{HSP}\left\{\left(L_{i}, \wedge, \vee, \text { all constants }\right)\right\} .
$$

From the fact that each diamond is a simple lattice (well known and easy to prove), we see that any lattice in $\mathscr{V}_{i}$ with $0 \neq 1$ (that is, any non-trivial lattice in $\mathscr{V}_{i}$ ) must have infinitely many constants appearing in it as distinct elements. Hence $\mathscr{V}_{i}$ contains no non-trivial finite algebras. Now we check that $\prod \mathscr{V}_{i}$ has the desired properties.

Fix a non-principal ultrafilter $\mathscr{F}$ on $\omega$; we will say that a property $p(i)$ of integers holds almost everywhere (a.e.) if and only if $\{i: p(i)$ is true $\} \in \mathscr{F}$. We will use $\mathscr{F}$ to define a congruence relation on $\prod L_{i} \in \prod \mathscr{V}_{i}$. First define

$$
\varphi: \prod L_{i} \rightarrow\{0,1\}
$$

via

$$
\varphi\left(a_{1}, a_{2}, \ldots\right)= \begin{cases}0, & \text { if for some } n, \text { height }\left(a_{i}\right)=n \text { a.e. } \\ 1, & \text { otherwise. }\end{cases}
$$

We wish to see that $\operatorname{ker} \varphi$ is a congruence relation on $L$, that is, that $\varphi(a)=\varphi(b)$ implies $\varphi(\alpha(a))=\varphi(\alpha(b))$ for every unary algebraic function $\alpha(x)$. Without loss of generality we may assume that $\varphi(a)=\varphi(b)=0$ and $\varphi(\alpha(a))=1$; from which we will deduce $\varphi(\alpha(b))=1$. 
By definition, $\alpha(a)=\left\langle\alpha_{i}\left(a_{i}\right)\right\rangle$ for some unary algebraic functions $\alpha_{i}$ on $L_{l}$. Our assumptions tell us that a.e. $\alpha_{i}\left(a_{i}\right)>z>a_{i}$; in particular, a.e.

$$
\alpha_{i}\left(a_{i}\right) \geqslant z>a_{i} \quad \text { with } z \vee \text {-prime }
$$

This last condition tells us (by an easy induction on the complexity of $\alpha_{i}(x)$ ) that in fact, a.e., $\alpha_{i}\left(a_{i}\right)$ is the least possible value of $\alpha_{i}(x)$, hence, a.e.,

$$
\alpha_{i}\left(b_{i}\right) \geqslant \alpha_{i}\left(a_{i}\right)
$$

And so $\varphi(\alpha(b))=1$.

And so $\prod \mathscr{V}_{i}$-operations may be defined on $\{0,1\}$ so that $\varphi$ becomes a homomorphism. It is easy to see from the definitions that each of these operations will be monotonic, and hence must be a polynomial in $0,1, \wedge, \vee$ (for example, by $G$. Birkhoff (1967, Theorem 5, p. 61)). Thus this homomorphic image generates a variety equivalent to that of bounded distributive lattices.

This proposition provides a counterexample to many of the assertions appearing in Neumann (1978), most notably 4.1, which claims that ' $\operatorname{Spec} \mathscr{V} \subseteq J$ ' is a strong Mal'tsev condition. It obviously also falsifies 3.1 , which claims that $\operatorname{Spec}\left(\prod \mathscr{V}_{i}\right)$ is the multiplicative closure of $\bigcup \operatorname{Spec} \mathscr{V}_{i}$ (a result which is true for finite products). The root of these troubles is Lemma 2.3, in whose proof that author has now found a mistake. The interested reader may consult the article and check that 6.1 and 7.1 are also false via this counterexample. (For 6.1, take $S=\{2\}$, $\mathscr{V}$ the variety of 'sets' (no operations) and $\mathscr{V}_{i} \subseteq \mathscr{V}$ the trivial variety defined by $x=y$.)

The finite product of varieties has a structure theory so strong that we can consider a finite product just as 'known' as its factors are (see, for example, Taylor (1973), p. 358 or $(1975$, p. 266). But clearly this is far from true for infinite products of varieties, even for their finite members. Calculations of infinite products (or merely some of their subvarieties, as we have done here) are rare in the literature; for a couple of examples see, for example, Taylor (1974), p. 348 and Bulman-Fleming and Taylor (1976), p. 191. Of course every proof that this or that Mal'tsev condition is not strong implicitly contains such a calculation; for references see Taylor (1973), p. 375. And so it would be interesting to see some more infinite products worked out in detail. We mention in this regard just one problem among many that occur naturally.

Problem 1.2. Do there exist varieties $\mathscr{V}_{i}$ such that $\operatorname{Spec} \mathscr{V}_{i}=\{1\}$ for each $i$, but $\prod \mathscr{V}_{i}$ contains a subvariety equivalent to 'sets' or 'pointed sets'? (That is, this subvariety has only constant operations.) 


\section{Proof of Theorem 2}

Let $A$ be a $(p+1)$-element set, and define $F: A^{p} \rightarrow A$ and $T: A^{3} \rightarrow A$ as follows:

$$
\begin{aligned}
F\left(a_{1}, \ldots, a_{p}\right) & =\left\{\begin{array}{l}
\text { the remaining member of } A \text { if } a_{1}, \ldots, a_{p} \text { are all distinct } \\
a_{1}, \text { otherwise. }
\end{array}\right. \\
T(a, b, c) & = \begin{cases}a & \text { if } a \neq b, \\
c & \text { if } a=b .\end{cases}
\end{aligned}
$$

(That is, $T$ is the ternary discriminator.) The quasiprimal algebra theory of Foster and Pixley (see Quackenbush (1979) for an exposition with references) tells us that the finite algebras in $\mathscr{V}=\operatorname{HSP}(A ; F, T)$ are the products of subalgebras of $(A ; F, T)$. Since $p$ is prime, we easily check that $\mathscr{V}$ contains no $p$-element algebras. The proof will be complete when we show that there exists a $p$-element algebra in $\mathscr{V}^{p-1}$. (In general, $\mathscr{V}^{n}$ denotes the variety $\supseteq \mathscr{V}$ which is defined by all $n$-variable identities of $\mathscr{V}-$ see Cohn (1963), p. 173 or Jónsson, McNulty and Quackenbush (1975).) For our algebra, we take $\mathfrak{B}=(B ; F, T)$ where $B$ is a $p$-element set, $F\left(b_{1}, \ldots, b_{n}\right)=b_{1}$ and $T$ is again the ternary discriminator. To see that $B \in \mathscr{V}^{p-1}$, it is enough by Cohn (1963, loc. cit.) to show that every $(p-1)$-generated subalgebra of $\mathfrak{B}$ is in $\mathscr{V}$; and this is obvious.

The example given in this proof can doubtless be greatly refined. We will extend it only to show the existence of a single Mal'tsev condition requiring $\aleph_{0}$ variables. A similar example appears at the end of Taylor (1972), but that example is really a generalized Mal'tsev condition (in the sense of Taylor (1973), p. 387), namely, it requires the use of a distinguished operation. Recall that $\mathscr{V}^{n}$ is defined in the proof just above.

THEOREM 2.1. There exists a Mal'tsev definable class $K$ such that for each $n$ there exists a variety $\mathscr{V} \in K$ with $\mathscr{V}^{n} \notin K$.

PrOOF. For each prime $p$, define $\mathfrak{A}_{p}$ to be the $(p+1)$-element algebra defined at the beginning of the above proof of Theorem 2, and define $\mathscr{V}_{p}$ to be HSP $\mathfrak{U}_{p}$. And, finally, define $\mathscr{W}_{p}$ to be the product variety $\mathscr{V}_{2} \times \mathscr{V}_{3} \times \ldots \times \mathscr{V}_{p}$. (Defined in Section 1 above. In some of our publications (1973), (1974), (1975) we used ' $\otimes$ ' for this kind of product, but we now feel it is reasonable to follow the example of Freyd (1966) and Neumann (1978) and reserve $\otimes$ for a different varietal product, which is closely related to tensor product of rings.)

$\mathscr{W}_{p}$ is defined by a single identity $\varphi_{p}$-see Baker (1977), McKenzie (1975), Padmanabhan and Quackenbush (1973) and Taylor (1979), 9.14. The sequence $\left(\varphi_{2}, \varphi_{3}, \varphi_{5}, \ldots\right)$ defines a Mal'tsev condition as described in our introduction; we 
take $K$ to be the class of varieties obeying this Mal'tsev condition; that is $\mathscr{V} \in K$ if and only if some $\varphi_{p}$ holds in $\mathscr{V}$ (with the $\varphi_{p}$-operations interpreted as $\mathscr{V}$-terms).

Since the sequence of primes $p$ is infinite, it will be enough to establish that $\mathscr{V}_{p} \in K$ but $\mathscr{V}_{p}^{p-1} \notin K$. The former fact is immediate from basic facts about product varieties. To see the latter, we assume that $\mathscr{V}_{p}^{p-1} \in K$ and derive a contradiction. First note that for some $q, \mathscr{V}_{p}^{p-1}$ satisfies $\varphi_{q}$; since $\varphi_{q}$ entails $\varphi_{q^{\prime}}$ for $q<q^{\prime}$ (this is why we took finite products), we may assume that $q>p$. (As in the earlier proof of this section) the theory of quasiprimal algebras (op. cit.) tells us that $\mathscr{V}_{2}, \mathscr{V}_{3}, \ldots, \mathscr{V}_{q}$ have no $q$-element algebras, and the theory of finite products of varieties (Taylor (1973), p. 358, (1975), p. 266) tells us that $\mathscr{W}_{q}$ has no $q$-element model. It will be enough to show that $\mathscr{V}_{p}^{p-1}$ does have a $q$-element model, for this will contradict the fact that $\mathscr{V}_{p}^{p-1}$ satisfies $\varphi_{q}$, the defining identity for $\mathscr{W}_{q}$. But obviously $(\{1, \ldots, q\}, F, T) \in \mathscr{V}_{p}^{p-1}$ for $F\left(a_{1}, \ldots, a_{p}\right)=a_{1}$ and $T$ the ternary discriminator.

\section{Spectral Mal'tsev conditions and Horn formulas}

Recently, Michael Morley has called to our attention both difficulties and successes associated with the problem of writing a Horn sentence $\varphi_{J}$ in pure equality theory which says, of an $n$-element set, 'either $n$ is infinite or $n \in J$ '. As above, $J$ is a cofinite set of integers containing 1 and closed under multiplication. It is easy to see the existence of an algorithm which proceeds from the finite complement of $J$ to yield $\varphi_{J}$, and Morley has provided a simpler algorithm than was known before.

In this section we will address Neumann's request for 'nice, explicit' conditions by providing an algorithm (3.3 below) which easily converts $\varphi_{J}$ into a Mal'tsev condition $\varphi_{1}, \varphi_{2}, \ldots$ for $J$ (described in the introduction). At the same time, we will give a new algorithm for constructing $\varphi_{J}$, which differs conceptually from previous ones in that it proceeds very simply from equational spectrum considerations. This algorithm for $\varphi_{J}$ will show that in some sense constructing $\varphi_{1}, \varphi_{2}, \ldots$ is just as hard as constructing $\varphi_{J}$, and so no improvement can really be made on our algorithm $\varphi_{J} \rightarrow \varphi_{1}, \varphi_{2}, \ldots$..

We give our main points in synopsis form, with detailed algorithms for 3.2 and 3.3 to follow.

3.1. There exists an algorithm to convert (the finite complement of) $J$ into a finite conjunction of identities $e_{J}$ whose spectrum is $J$. Such an algorithm may be found in McKenzie (1975); the number of steps required, and the length of $e_{J}$, are no more than $k n^{2}$, where $n=$ maximum $m \notin J$. 
3.2. We will give an algorithm to convert any identities $e_{J}$ with spectrum $J$ to a Horn sentence $\varphi_{J}$ as above. The length of $\varphi_{J}$ is linear, and the number of steps required is quadratic, in the length of $e_{\mathrm{J}}$.

3.3. We will give an algorithm to convert any $\varphi_{J}$ into a Mal'tsev condition $\varphi_{1}, \varphi_{2}, \ldots$ for $J$, in which each $\varphi_{i}$ has spectrum $J$. More precisely, in time linear in $m=$ length $\left(\varphi_{J}\right)$, our algorithm produces a simple algorithm $A_{J}$ for the sequence $\left\{\varphi_{i}\right\}$. (To produce an individual $\varphi_{i}$ takes $K m^{i}$ steps-but this means very little, since the individual $\varphi_{i}$ have no invariant meaning in this problem; and since individual $\varphi_{i}$ may be repeated or deleted at will, even their growth rate has no real significance.)

3.4. If $\varphi_{1}, \varphi_{2}, \ldots$ is any Mal'tsev condition for $J$, then, from some $N$ onward, each $\varphi_{n}$ has spectrum $J$. This is an easy consequence of 3.1 ; moreover such an $N$ is recursive in (a Turing machine for) the sequence $\varphi_{1}, \varphi_{2}, \ldots$ Our contention that knowing $\varphi_{1}, \varphi_{2} \ldots$ is probably no easier than knowing $\varphi_{J}$ rests on the observation that, as soon as we know this $N$, we can recover $\varphi_{J}$ from $\varphi_{N}$, using 3.2. While one can easily imagine silly ways to make $N$ very large and difficult to compute, reasonable procedures-which get right down to business-generally have $N$ small and apparent. In fact $N=1$ both in the general case given in 3.3 above and in the special case $(J=\omega-\{2\})$ given on p. 383 of Taylor (1973).

For the definition and basic theory of Horn formulas, see, for example, Chang and Keisler (1973), Sections 6.2, 6.3, where there is a proof of the existence of $\varphi_{J}$. For our first algorithm, the reader does not need the full definition, only our assurance that the constructed $\varphi_{J}$ is Horn.

ALGORITHM 3.2 from $e_{J}$ to $\varphi_{J}$. We know that $e_{J}$ has the form

$$
\forall x_{1} \ldots x_{s} \bigwedge_{k=1}^{t} \sigma_{k}=\tau_{k},
$$

where $\sigma_{k}$ and $\tau_{k}$ are terms in $x_{1}, \ldots, x_{s^{*}}$ Take one new variable $y_{\alpha}$ for each subterm $\alpha$ of each $\sigma_{i}$ and $\tau_{i}$. For each $i=1, \ldots, s$, we take the formula

$$
x_{i}=y_{\sigma_{i}} \text {. }
$$

If $\alpha=F\left(\alpha_{1}, \ldots, \alpha_{p}\right), \beta=F\left(\beta_{1}, \ldots, \beta_{p}\right)$, then we take the 'basic Horn formula':

$$
\left(\bigwedge_{i=1}^{p} y_{\alpha_{i}}=y_{\beta_{i}}\right) \rightarrow y_{\alpha}=y_{\beta} .
$$

And, finally, for $k=1, \ldots, t$ we take

$$
y_{\sigma_{k}}=y_{\tau_{k}}
$$


(with $\sigma_{k}, \tau_{k}$ as given in $\varphi_{n}$ ). The required $\varphi_{J}$ is then

$$
\forall x_{1} \ldots x_{s} \exists \ldots y_{\sigma} \ldots \bigwedge_{q=1}^{N} H_{q},
$$

where $H_{1}, \ldots, H_{N}$ are the formulas given in (3.5), (3.6) and (3.7). This completes our description of the algorithm; to see that the constructed $\varphi_{J}$ is as desired, we will need this lemma:

LeMma. For $\varphi_{J}$ as defined in the algorithm, and any finite set $X$,

$$
|X| \in J \text { if and only if } X \| \varphi_{J} .
$$

Proof. If $|X| \in J$, then $X$ is the universe of an algebra obeying $e_{J}$. We simply use the operations defined on this algebra to find all the $y_{\sigma}$ so that $(3.5),(3.6)$ and (3.7) all become true.

Conversely, if $X \Vdash \varphi_{J}$, then (3.5), (3.6) and (3.7) obviously provide a way of unambiguously defining the operations of $e_{J}$ on $X$ so that $e_{J}$ becomes true.

AlgorithM 3.3 from $\varphi_{J}$ to $\varphi_{1}, \varphi_{2}, \ldots$ We shall assume that $\varphi_{J}$ is given in the form

$$
\varphi_{J}=\forall x_{1} \ldots x_{s} \exists y_{1} \ldots y_{t} \bigwedge_{i=1}^{n}\left[\left(\bigwedge_{j=1}^{m} \alpha_{i j}=\beta_{i j}\right) \rightarrow \gamma_{i}=\delta_{i}\right] \text { ? }
$$

where each $\alpha, \beta, \gamma$ and $\delta$ is one of the $x$ 's or one of the $y$ 's. (The existence of this form follows of course from the lemma just above, or from traditional theorems in model theory-for example, Exercise 6.3.4 on p. 367 of Chang and Keisler (1973); in any case an arbitrary Horn sentence in pure equality theory is easily converted to this form.) We next claim that it is easy to convert $\varphi_{J}$ so that each $\gamma$ and each $\delta$ is one of the $x$ 's. This is an easy inductive argument based on the observation that $\varphi_{J}$ is obviously equivalent to

$$
\begin{aligned}
\forall x_{0} x_{1} \ldots x_{s} \exists y_{1} \ldots y_{t} \bigwedge_{i=2}^{n}\left[\left(\bigwedge_{j=1}^{m} \alpha_{i j}=\beta_{i j}\right) \rightarrow \gamma_{i}=\delta_{i}\right] \\
\wedge\left[\left(x_{0}=\gamma_{1}\right) \wedge\left(\bigwedge_{j=1}^{m} \alpha_{i j}=\beta_{i j}\right) \rightarrow x_{0}=\delta_{1}\right]
\end{aligned}
$$

A standard compactness argument now allows one to prove that for a variety $\mathscr{r}$, Spec $\mathscr{V} \subseteq J$ if and only if for some terms $F_{k}^{\lambda}\left(x_{1}, \ldots, x_{s}\right)(1 \leqslant \lambda \leqslant N, 1 \leqslant k \leqslant t)$, $\mathscr{V}$ satisfies

$$
\forall x_{1} \ldots x_{s} \bigvee_{\lambda=1}^{N} \bigwedge_{i=1}^{n}\left[\left(\bigwedge_{j=1}^{m} \alpha_{i j}^{\lambda}=\beta_{i j}^{\lambda}\right) \rightarrow \gamma_{i}=\delta_{i}\right],
$$

where $\alpha_{i j}^{\lambda}$ is obtained from $\beta_{i j}$ by leaving each $x$ alone, and converting each $y_{k}$ to $F_{k}^{\lambda}\left(x_{1}, \ldots, x_{s}\right)$. (We omit a detailed proof.) An application of the distributive law 
for $\wedge$ and $\vee$, together with the fact that $\mathscr{V}$ is closed under formation of products, tells us that $\operatorname{Spec} \mathscr{V} \subseteq J$ if and only if for some terms $F_{k}^{\lambda}$ as above and some $\theta: n^{N} \rightarrow n, \mathscr{V}$ satisfies

$$
\forall x_{1} \ldots x_{s} \bigwedge_{\gamma \in n^{N}}\left[\left(\bigwedge_{\lambda=1}^{N} \bigwedge_{j=1}^{m} \alpha_{\gamma(\lambda), j}^{\lambda}=\beta_{\gamma(\lambda), j}^{\lambda}\right) \rightarrow \gamma_{\theta(\gamma)}=\delta_{\theta(\gamma)}\right]
$$

And this final condition is easily converted into a Mal'tsev condition (see, for example, p. 397 of Taylor (1973)).

\section{Varieties of varieties}

On p. 115 of Neumann (1978), Neumann defined a class $K$ of varieties to be a variety of varieties if and only if $K$ is closed under formation of product varieties, subvarieties and images of pure forgetful functors. He then asks whether the lattice of varieties of varieties is 'quite sparse'. Here we will prove the easy Theorem 3 of the introduction, which states that there are $2^{\aleph_{0}}$ varieties of varieties.

Proof of Theorem 3. Isbell (1970) and Vaughan-Lee (1970) established that there are $2^{N_{0}}$ varieties of monoids (semigroups with a unit element in the similarity type). (Also see Biryukov (1965) and Evans (1968).) It will thus be enough to give, for each variety $\mathscr{M}$ of monoids, a variety $K_{\mathscr{M}}$ of varieties such that distinct $\mathscr{M}$ 's yield distinct $K_{\mathscr{M}}$ 's.

If $\mathscr{V}$ is any variety (of any similarity type), then the unary operations of $\mathscr{V}$ form a monoid $M(\mathscr{V})$, in which the product $F G$ is given by $F G(x)=F(G(x))$. (In fact $M(\mathscr{V})$ is simply the degree-1 part of the clone-see, for example, Taylor (1973), pp. 361-364-or the endomorphism monoid of the object 1 in the 'algebraic theory' of $\mathscr{V}$-see, for example, (op. cit. p. 391). We now define

$$
K_{\mathscr{M}}=\{\mathscr{V}: M(\mathscr{V}) \in \mathscr{M}\} .
$$

It is routine to check that $K_{\mathscr{M}}$ is a variety of varieties. Now if $\mathscr{M} \neq \mathscr{N}$, we may without loss of generality find a monoid $M \in \mathscr{M}-\mathscr{N}$. Let $\mathscr{V}_{M}$ be the variety of all ' $M$-sets', that is $\mathscr{V}_{M}$ has one unary operation $f$ for each $f \in M$, subject to the laws

$$
\begin{gathered}
\bar{f}(\bar{g}(x))=\bar{f} \bar{g}(x), \\
\overline{1}(x)=x .
\end{gathered}
$$

One easily checks that $M\left(\mathscr{V}_{M}\right)=M$, and hence $\mathscr{V}_{M} \in K_{\mathcal{M}}-K_{\mathscr{V}}$. 


\section{References}

K. Baker (1977), 'Finite equational bases for finite algebras in a congruence-distributive equational class', Advances in Math. 24, 207-243.

J. T. Baldwin and J. Berman (1977), 'A model-theoretic approach to Malcev conditions', $J$. Symbolic Logic 42, 277-288.

G. Birkhoff (1967), Lattice theory 3rd ed. (Amer. Math. Soc. Colloq. Publications, 25, New York).

A. P. Biryukov (1965), 'On infinite sets of identities in semigroups', Algebra i Logika 4, 31-32.

S. Bulman-Fleming and W. Taylor (1976), 'Union-indecomposable varieties', Colloq. Math. 35, 189-199.

C. C. Chang and H. J. Keisler (1973), Model Theory (Studies in Logic and the Foundations of Mathematics, 73, North-Holland, Amsterdam, 1973).

P. M. Cohn (1965), Universal Algebra (Harper's Series in Modern Mathematics, Harper and Row, New York, 1965).

T. Evans (1968), 'The number of semigroup varieties', Quart. J. Math. (Oxford) (2) 19, 335-336.

P. Freyd (1966), 'Algebra valued functors in general, and tensor products in particular', Colloq. Math. 14, 89-106.

G. Grätzer (1968), Universal algebra (The University Series in Higher Mathematics, Van Nostrand, Princeton, 1968).

J. R. Isbell (1970), 'Two examples in varieties of monoids', Proc. Cambridge Philos. Soc. 68, 265-266.

B. Jónsson, G. McNulty and R. W. Quackenbush (1975), 'The ascending and descending varietal chains of a variety', Canad. J. Math. 27, 25-32.

R. McKenzie (1975), 'On spectra, and the negative solution of the decision problem for identities having a finite non-trivial model', J. Symbolic Logic 40, 186-196.

W. D. Neumann (1974), 'On Malcev conditions', J. Austral. Math. Soc. 17, 376-384.

W. D. Neumann (1978), 'Mal'cev conditions, spectra and Kronecker products', J. Austral. Math. Soc. Ser. A 25, 103-117.

R. Padmanabhan and R. W. Quackenbush (1973), 'Equational theories of algebras with distributive congruences', Proc. Amer. Math. Soc. 41, 373-377.

R. W. Quackenbush (1979), 'Primal algebras', an appendix to the forthcoming new edition of G. Grätzer (1968) (to appear).

W. Taylor (1972), 'Fixed points of endomorphisms', Algebra Universalis 2, 74-76.

W. Taylor (1973), 'Characterizing Mal'cev conditions', Algebra Universalis 3, 351-397.

W. Taylor (1974), 'Uniformity of congruences', Algebra Universalis 4, 342-360.

W. Taylor (1975), 'The fine spectrum of a variety', Algebra Universalis 5, 263-303.

W. Taylor (1978), 'Miscellaneous results on Mal'tsev conditions', Notices Amer. Math. Soc. 25, A-581.

W. Taylor (1979), review of W. D. Neumann (1978), MR., 58

W. Taylor (1979a), 'Equational logic', Houston J. Math. Survey volume 1979, ii +83 pp. An abridgement is to appear in the forthcoming new edition of G. Grätzer (1968).

M. R. Vaughan-Lee (1970), 'Uncountably many varieties of groups', Bull. London Math. Soc. 2, 280-286.

Department of Mathematics

University of Colorado

Boulder, Colorado 80309

USA 